\title{
MicroRNA expression profiles of esophageal cancer
}

\author{
Andrew Feber, PhD, ${ }^{a}$ Liqiang Xi, MD, ${ }^{\text {a }}$ James D. Luketich, $\mathrm{MD}^{\mathrm{c}}$ Arjun Pennathur, MD, ${ }^{\mathrm{c}}$ Rodney J. Landreneau, MD, ${ }^{\mathrm{c}}$ \\ Maoxin $\mathrm{Wu}, \mathrm{MD},{ }^{\mathrm{a}}$ Scott J. Swanson, MD, Tony E. Godfrey, PhD, ${ }^{\mathrm{a}}$ and Virginia R. Litle, MD ${ }^{\mathrm{b}}$
}

From the Department of Pathology and the Division of Thoracic Surgery, Department of Cardiothoracic Surgery, ${ }^{\mathrm{b}}$ Mount Sinai Medical Center, New York, NY; and the Heart, Lung and Esophageal Surgery Institute, University of Pittsburgh Medical Center, Pittsburgh, Pa. ${ }^{\mathrm{C}}$

Received for publication May 4, 2007; revisions received Aug 12, 2007; accepted for publication Aug 15, 2007.

Address for reprints: Virginia R. Litle, MD, Division of Thoracic Surgery, Mount Sinai Medical Center, 1190 Fifth Ave, Box 1028, New York, NY 10029 (E-mail: virginia.litle@mountsinai.org).

J Thorac Cardiovasc Surg 2008;135:255-60 $0022-5223 / \$ 34.00$

Copyright $\odot 2008$ by The American Association for Thoracic Surgery

doi:10.1016/j.jtcvs.2007.08.055
Objective: Expression of microRNAs by array analysis provides unique profiles for classifying tissues and tumors. The purpose of our study was to examine microRNA expression in Barrett esophagus and esophageal cancer to identify potential markers for disease progression.

Methods: MicroRNA was isolated from 35 frozen specimens (10 adenocarcinoma, 10 squamous cell carcinoma, 9 normal epithelium, 5 Barrett esophagus, and 1 high-grade dysplasia). MicroRNA expression was analyzed with Ambion bioarrays (Ambion, Austin, Tex) containing 328 human microRNA probes.

Results: Unsupervised hierarchic clustering resulted in four major branches corresponding with four histologic groups. One branch consisted of 7 normal epithelium samples and 1 squamous cell carcinoma sample. The second branch consisted of 7 squamous cell carcinoma samples and 1 normal epithelium sample. The third branch contained 4 Barrett esophagus samples and 1 squamous cell carcinoma sample. The fourth contained all the adenocarcinoma samples and 1 sample each of Barrett esophagus, normal epithelium, squamous cell carcinoma, and high-grade dysplasia. Supervised classification with principal component analysis determined that the normal epithelium samples were more similar to the squamous cell carcinoma tumors, whereas the Barrett esophagus samples were more similar to adenocarcinoma. Pairwise comparisons between sample types revealed microRNAs that may be markers of tumor progression. Both mir_203 and mir_205 were expressed 2- to 10-fold lower in squamous cell carcinoma and adenocarcinomas than in normal epithelium. The mir_21 expression was 3- to 5-fold higher in both tumors than in normal epithelium. Prediction analysis of microarray classified 3 Barrett esophagus samples as Barrett esophagus, 1 as adenocarcinoma, and 1 as normal epithelium.

Conclusion: Expression profiles of miRNA distinguish esophageal tumor histology and can discriminate normal tissue from tumor. MicroRNA expression may prove useful for identifying patients with Barrett esophagus at high risk for progression to adenocarcinoma.

$\mathrm{E}$ sophageal adenocarcinoma (AC) has the fastest increasing incidence of any solid tumor in the United States, ${ }^{1}$ and the 5 -year survival for this disease remains dismal, ranging from $14 \%$ to $22 \% .^{2-4}$ Whereas the incidence of esophageal squamous cell carcinoma (SCC) has been declining or has remained constant in the United States, that of esophageal AC has increased more than $300 \%$ in the past 30 years and continues to rise. ${ }^{1,5-7}$ The most common risk factor for AC is chronic gastroesophageal reflux disease, which is associated with an approximately 16-fold increased risk of $\mathrm{AC}$ and occurs in as many as $60 \%$ of patients in whom this tumor is diagnosed. ${ }^{8}$ Gastroesophageal reflux disease causes inflammation in the distal esophagus, resulting in development of the premalignant lesion known as Barrett esophagus (BE) or intestinal metaplasia, ${ }^{9}$ and the presence of BE confers a $0.5 \%$ per patient-year risk of development of AC. ${ }^{10,11}$ The progression of BE to AC develops through established histologic changes: intestinal metaplasia (BE) to low-grade dysplasia to highgrade dysplasia (HGD) to AC. HGD represents an unstable epithelium that is a marker for concurrent occult carcinoma or for subsequent development of carcinoma, with 

Abbreviations and Acronyms
$\mathrm{AC}=$ adenocarcinoma
$\mathrm{BE} \quad=$ Barrett esophagus
HGD = high-grade dysplasia
miRNA $=$ microRNA
NSE = normal squamous epithelium
$\mathrm{PAM}=$ prediction analysis of microarray
SCC = squamous cell carcinoma

such risk ranging from $25 \%$ to $80 \%$ in multiple series. ${ }^{12-15}$ The current standard of care for patients with HGD and low operative risk factors is esophagectomy. If the patient chooses not to undergo surgical resection or is deemed a poor surgical candidate, alternatives such as endomucosal resection, photodynamic therapy, or other local therapies may be proposed. ${ }^{16-18}$ Patients are then advised to undergo endoscopies and multiple random biopsies every three months. Similarly, patients with low-grade dysplasia are advised to undergo serial endoscopies, albeit less frequently, to monitor for development of HGD. ${ }^{19}$

Dysplasia alone as a marker of malignant progression is marred by pathologists' differential interpretation of degrees of dysplasia. ${ }^{20}$ Because only a subset of dysplastic lesions progress to cancer, the challenge lies in substratification of biopsy samples according to the probability of malignancy development. If molecular markers of premalignancy can be identified on small pieces of esophageal tissue obtained endoscopically, this could lead to improved early detection and provide objective criteria for selection of patients who might benefit from aggressive surgical treatment.

MicroRNAs (miRNAs), a class of small, noncoding RNA molecules first reported on in $1993,{ }^{21}$ are posttranscriptional regulators of gene expression that have been shown to be involved in cell differentiation, proliferation, and apoptosis. Furthermore, it has recently been demonstrated that some miRNAs may function as either oncogenes or tumor suppressor genes (oncomirs). ${ }^{22}$ Altered expression of oncomirs has now been found in a variety of human tumors including lung, breast, and prostate cancers, ${ }^{23-27}$ and recent reports demonstrate a role for miRNA expression in disease progression and outcome. ${ }^{24,26}$ Although protein-coding gene expression studies have shown differences between normal squamous epithelium (NSE) in the esophagus and BE and between $\mathrm{BE}$ and $\mathrm{AC},{ }^{28,29}$ only one recent report on 7 patients has determined miRNA expression in esophageal lesions. ${ }^{30}$

We hypothesized that unique miRNA expression profiles exist to distinguish normal esophagus, BE, esophageal dysplasia, and esophageal cancer. Furthermore, because miRNA expression is associated with differentiation, specific miRNAs may become deregulated in the progression from $\mathrm{BE}$ to dysplasia to AC. Identification of these differences in miRNA expression could then discriminate patients who have a high risk for progression to cancer and who therefore need to be followed up more closely or treated more aggressively.

\section{Materials and Methods Specimens}

Esophageal specimens were obtained from patients undergoing esophagectomy for malignant disease during the years 1999 through 2005 at the University of Pittsburgh Medical Center and Mount Sinai Medical Center. This study involving human tissue was approved by the institutional review boards at both institutions. Histologic confirmation of the esophageal diagnosis was performed by the pathologist at the Mount Sinai Medical Center (M.W.).

Thirty-five frozen specimens were chosen for analysis. There were $10 \mathrm{AC}$ specimens, $10 \mathrm{SCC}$ specimens, $5 \mathrm{BE}$ specimens, 1 HGD lesion specimen, and 9 NSE specimens. NSE specimens were obtained from patients undergoing esophagectomy for HGD or cancer and were taken from as far from the malignancy as possible.

\section{Isolation of miRNA}

Enriched miRNA was isolated from tissue specimens with the mirVana miRNA Isolation Kit (Ambion Inc, Austin, Tex), which allows capture of both enriched miRNA and larger RNA species separately. Both the enriched miRNA and large RNA were quantified with a NanoDrop spectrophotometer (NanoDrop Technology, Wilmington, Del), and integrity of the large RNA fraction was determined with an Agilent Bioanalyzer (Agilent Technologies, Palo Alto, Calif) as a surrogate for miRNA quality control. The miRNA samples were only used in microarray experiments if the RNA integrity number for the large RNA from the same specimen was at least 6 .

\section{Labeling and Hybridization of miRNA, Scanning, and Data Processing}

Samples of miRNA from 35 esophageal specimens were labeled with the mirVana miRNA Labeling Kit (Ambion) according to the manufacturer protocol. The Cy5-labeled miRNA was then hybridized at $42^{\circ} \mathrm{C}$ for 16 hours on mirVana miRNA Bioarrays (Ambion), which contain 796 probes representing duplicate spots for each of 287 human miRNAs, 65 mouse and rat miRNAs, 33 Ambionpredicted miRNAs, and 4 positive controls (20 replicate spots for each positive control). The slides were then washed and scanned with an Axon 4000B (Molecular Devices Corporation, Sunnyvale, Calif) scanner at $100 \%$ power and a photomultiplier tube gain of 750. The microarray images were analyzed with Genepix PRO (version 5.1; Molecular Devices), and normalization was performed with CyclicLoess ${ }^{31}$ in R program downloaded from the Bioconductor open source project (http://www.bioconductor.org). Unsupervised hierarchic clustering, differentially expressed gene analysis among the groups, and prediction analysis of microarray (PAM) were performed with the National Cancer Insitute BRB-ArrayTools Version 3.4 (BRB-ArrayTools Version 3.4; Biometrics Research Branch, National Cancer Institute, Bethesda, Md). For differentially expressed gene analysis among four histologic types (AC, SCC, NSE, BE), class comparison between groups of arrays was performed with a random-variance $F$ test. Genes were considered statistically significant if their $P$ value was less than .001 . A stringent significance threshold was used to limit the number of false-positive findings. This tool also used the multivariate permutation test to 
provide $90 \%$ confidence that the false discovery rate was less than $10 \%$. The false discovery rate is the proportion of the list of genes claimed to be differentially expressed that are expected to be false positives. For PAM, 10-fold cross validation was performed with samples of four histologic types (AC, SCC, NSE, and $\mathrm{BE}$ ). The comparison of PAM with histologic examination was facilitated by transforming the continuous PAM output into binary data with a probability of 0.5 as the cut off. Principal component analysis was performed with Partek Genomics Suite software (Partek, Inc, St Louis, Mo).

\section{Results}

Sample Classification With Unsupervised Hierarchic Clustering

We analyzed miRNA expression in $10 \mathrm{AC}, 10 \mathrm{SCC}, 5 \mathrm{BE}, 1$ HGD, and 9 NSE samples. Unsupervised hierarchic clustering of samples with all miRNAs was able to separate NSE, SCC, BE, and AC into four major groups with a few exceptions (Figure 1, A). SCC sample MS305 was exceptional, with an miRNA pattern clustered in the AC group. The histologic type of MS305 was AC according to frozen-section analysis but was later interpreted as SCC on the fixed-tissue slides for final pathologic study. After the miRNA profile was obtained, the fixed-tissue slides were reviewed again by a pathologist at University of Pittsburgh Medical Center, who determined that it was a basaloid SCC with focally undifferentiated areas that could look like a poorly differentiated AC.
Significantly Differentially Expressed miRNAs

In addition to unsupervised sample classification, we also performed analysis to identify miRNAs that were differentially expressed among different histologic groups. Our results indicated that 13 human miRNAs are significantly differentially expressed among AC, SCC, and NSE (Table 1). The miRNAs miR_194, miR_192, and miR_200c are significantly upregulated in AC but not in SCC. Relative to NSE, miR_342 is differentially expressed in SCC but not AC. Others, including miR_21, miR_205, miR_203, and miR_93, are differentially expressed between tumor and normal tissue but not necessarily between the two tumor histologic types.

\section{Supervised Sample Classification With Principal Component Analysis}

Principal component analysis was performed with significantly differentially expressed genes (described previously) on the 35 esophageal specimens. As with the cluster analysis, principal component analysis was able to separate the four main types of samples (NSE, AC, SCC, and BE) into distinct groups. This analysis also provided a clear visual representation of similarity between the histologic types (Figure 1, B). The NSE and SCC samples were more similar to each other than to the AC samples. The BE samples sat between the AC and NSE samples, and the single HGD specimen had a miRNA expression profile similar to the AC sample.
Dendrogram for clustering experiments, using centered correlation and average linkage.

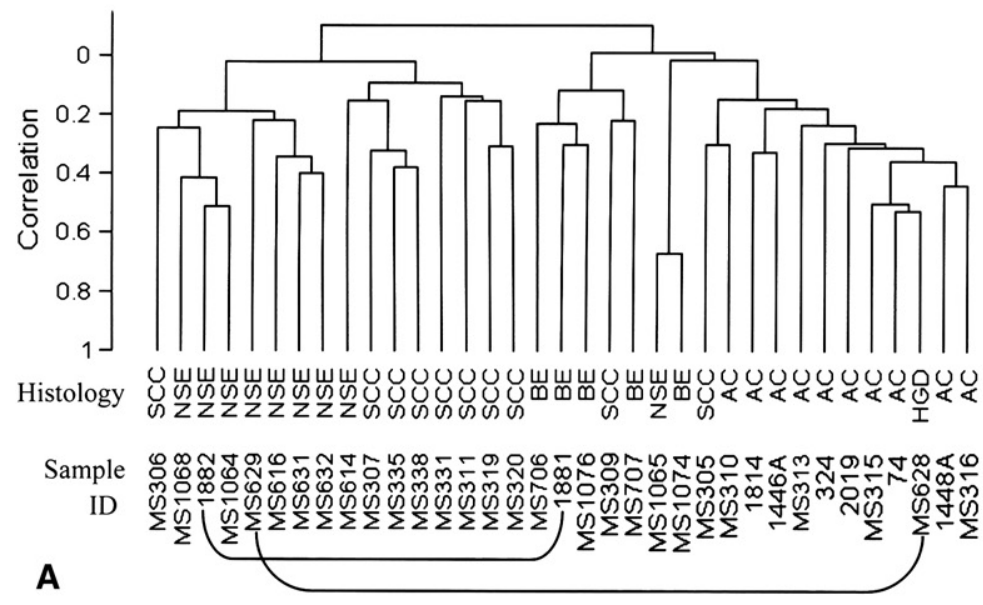

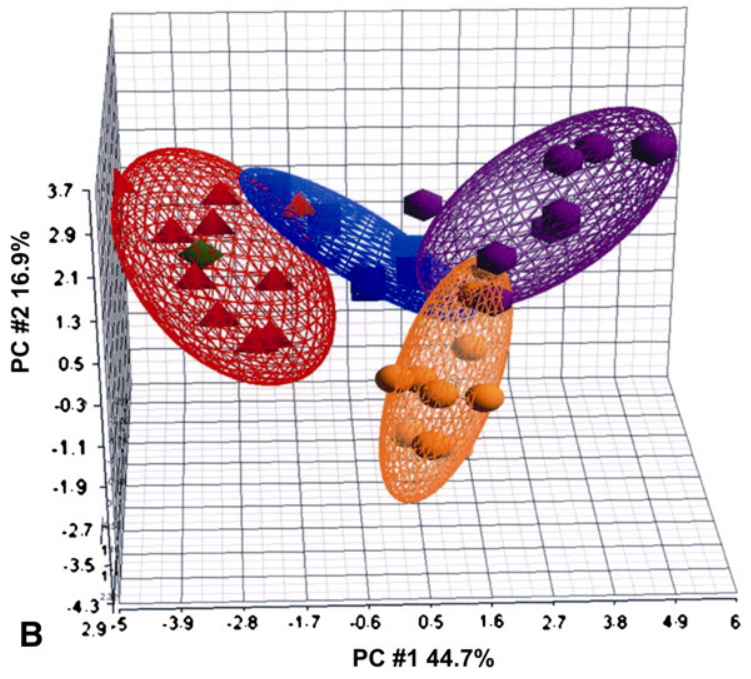

Figure 1. MicroRNA expression patterns distinguish esophageal adenocarcinoma (AC), squamous cell carcinoma (SCC), normal squamous epithelium (NSE), Barrett esophagus (BE), and high-grade dysplasia (HGD). A, Unsupervised hierarchic clustering of esophageal specimens with all expressed miRNAs. Linked samples (1882/1881 and MS629/ MS628) were obtained from the same patient. B, Principal component analysis mapping based on 14 miRNAs that exhibit differential expression between adenocarcinoma (red triangle), squamous cell carcinoma (yellow circle), normal squamous epithelium (purple hexagon), Barrett esophagus (blue square), and high-grade dysplasia (green diamond). 
TABLE 1. Differentially expressed microRNAs among four histologic types (adenocarcinoma, squamous cell carcinoma, Barrett esophagus, and normal squamous epithelium)

\begin{tabular}{llllll}
\hline & \multicolumn{5}{c}{ Fold change } \\
\cline { 2 - 5 } \multicolumn{1}{c}{ MicroRNA } & AC vs BE AC vs NSE SCC vs NSE AC vs SCC & FDR \\
\hline hsa_miR_192 & 1.7 & 3.9 & 0.9 & 4.4 & $<1 \mathrm{e}-07$ \\
hsa_miR_194 & 2 & 4.7 & 0.8 & 5.6 & $<1 \mathrm{e}-07$ \\
mmu_miR_192 & 1.4 & 3.0 & 0.9 & 3.4 & $<1 \mathrm{e}-07$ \\
hsa_miR_21 & 3.7 & 5.2 & 2.9 & 1.8 & 0.0003384 \\
hsa_miR_27b & 0.7 & 0.4 & 0.6 & 0.7 & 0.0003384 \\
hsa_miR_205 & 0.9 & 0.1 & 0.5 & 0.3 & 0.0005452 \\
hsa_miR_203 & 0.9 & 0.2 & 0.4 & 0.6 & 0.0009346 \\
hsa_miR_200c & 1.9 & 1.9 & 0.8 & 2.4 & 0.0014706 \\
hsa_miR_342 & 0.8 & 0.8 & 1.8 & 0.4 & 0.0014706 \\
hsa_let_7c & 1.1 & 0.6 & 0.5 & 1.2 & 0.006298 \\
hsa_miR_93 & 1.3 & 1.4 & 1.3 & 1.1 & 0.0107505 \\
hsa_miR_125b & 0.5 & 0.5 & 0.8 & 0.6 & 0.0107505 \\
hsa_miR_100 & 0.8 & 0.5 & 0.7 & 0.7 & 0.0112511 \\
hsa_miR_152 & 1 & 0.8 & 1.1 & 0.8 & 0.0147661
\end{tabular}

$A C$, Adenocarcinoma; $B E$, Barrett esophagus; $N S E$, normal squamous epithelium; $S C C$, squamous cell carcinoma; $F D R$, false discovery rate.

\section{Sample Classification With PAM}

PAM identified a set of miRNAs, the expression of which was able to classify samples accurately according to histologic type. All $10 \mathrm{AC}$ and $10 \mathrm{SCC}$ samples were accurately classified into the correct histologic groups, whereas 2 NSE samples were classified incorrectly, 1 as BE and 1 as SCC. Of the $5 \mathrm{BE}$ samples, 3 were classified as BE, 1 was called $\mathrm{AC}$, and the other was classified as NSE. With the same miRNA classifier, the single HGD sample was determined to be more similar to AC than to the other three histologic types. These data are shown in Table 2.

\section{Discussion}

The incidence of esophageal cancer has increased more than $300 \%$ in the United States since the 1970s, and the overall cure rate is dismal and failing to improve. BE, the earliest known premalignant lesion for this cancer, is associated with a risk of cancer of $0.5 \%$ per patient-year. ${ }^{11,32}$ Current national guidelines from the American College of Gastroenterology include serial endoscopies and random biopsies to identify dysplasia and cancer in this small but high-risk population of patients with reflux. ${ }^{10,11}$ In addition to the potential sampling error with random endoscopic biopsies, the interpretation of dysplasia is subject to interobserver variability. The best clinical management of these patients is therefore still limited by our current diagnostic approach and our understanding of the disease process.

Global gene expression (messenger RNA) profiling of BE and esophageal cancer has previously been used to identify differentially expressed genes in BE relative to esophageal
TABLE 2. Prediction analysis of microarray classification of samples according to miRNA expression

\begin{tabular}{lcrccc}
\hline & & \multicolumn{4}{c}{ Classification } \\
\cline { 3 - 6 } Histologic type & No. of samples & AC & SCC & NSE & BE \\
\hline AC & 10 & 10 & 0 & 0 & 0 \\
SCC & 10 & 0 & 10 & 0 & 0 \\
NSE & 9 & 0 & 1 & 7 & 1 \\
BE & 5 & 1 & 0 & 1 & 3 \\
HGD & 1 & 1 & 0 & 0 & 0
\end{tabular}

Training was performed on all samples except the single high-grade dysplasia with 10 -fold internal cross-validation. The high-grade dysplasia sample classification was then predicted on the basis of the training analysis but forced into one of the histologic types used in the training. $A C$, Adenocarcinoma; $S C C$, squamous cell carcinoma; NSE, normal squamous epithelium; $B E$, Barrett esophagus; $H G D$, high-grade dysplasia.

AC. ${ }^{33}$ In nonesophageal tissues, miRNA expression profiling has been shown to provide more accurate classification of tissue and tumor types than global messenger RNA expression profiles. ${ }^{23,34}$ For example, differential miRNA expression has been used to discriminate lung cancer from benign lung tissue, lung $\mathrm{AC}$ from $\mathrm{SCC},{ }^{26}$ and malignant from benign tissues in the prostate and thyroid. ${ }^{27,35}$ In addition, miRNA plays a role in lineage-specific tissue differentiation and as such is particularly good at identifying the source of poorly differentiated tumors of unknown origin. ${ }^{23}$ We therefore hypothesized that (1) tumor-specific miRNA expression profiles exist for malignant and normal esophageal tissues and (2) miRNA expression profiles may be associated with progression from $\mathrm{BE}$ to $\mathrm{AC}$.

To test our hypotheses, we used miRNA expression arrays from Ambion to determine the miRNA profiles for esophageal NSE, BE, an HGD lesion, and the two primary types of esophageal cancer, AC and SCC. In this series we demonstrated that miRNA expression profiles distinguish different esophageal tissue types and also discriminate malignant from normal esophageal tissue. Furthermore, the NSE and SCC samples were much more similar to each other than to the AC samples. Similarly, BE and AC miRNA expression profiles were more similar to each other than to tissues of squamous origin (either benign or malignant). In light of our current understanding of the development of esophageal $\mathrm{AC}$, this makes good biologic sense, because $\mathrm{AC}$ is believed to arise from Barrett columnar epithelium and not from squamous epithelium.

In addition to identifying differential expression of particular miRNAs in NSE, BE, AC, and SCC of the esophagus, we also used the miRNA data to predict into which groups the BE specimens would be genotyped on the basis of miRNA expression rather than histologic examination. This analysis identified possible subtypes of miRNA expression within the 5 BE samples: 1 with a "normal" profile, 1 with an "AC" profile, and 3 with BE-specific miRNA expression. 
These results support future studies in a larger sample of patients with BE undergoing cancer follow-up to examine the hypothesis that miRNA expression may distinguish a subgroup of BE patients most likely to have esophageal cancer develop.

Finally, there are reports in the literature associating some of the differentially expressed miRNAs reported on here with other cancers. For example, miR_21 is upregulated in both esophageal AC and SCC, and this has also been reported in tumors of the breast, lung, prostate, colon and stomach, as well as in glioblastomas. ${ }^{25,26,35,36}$ In addition, miR_192 and miR_203 expressions were upregulated in lung cancer tissue relative to normal lung, ${ }^{36}$ and inhibition of miR_192 has been shown to downregulate cell growth in a lung AC cell line. ${ }^{38}$ In our data, we saw higher expression of miR_192 in esophageal AC but lower expression of miR_203 in both cancer types relative to NSE of the esophagus.

\section{Conclusions}

The differential miRNA expressions of BE and esophageal AC may lead to identification of specific markers for progression, additional molecular classification of dysplastic lesions, and a better understanding of the biology of progression of $\mathrm{BE}$ and dysplasia. Improvement in survival of patients with $\mathrm{AC}$ will most likely result from new therapies that are based on an increased understanding of the tumor biology and identification of biomarkers for earlier detection.

We thank Dr José Walewski for the use of the Axon scanner and Dr Alyssa Krasinskas for reviewing pathology on ambiguous specimens.

\section{References}

1. Pohl H, Welch HG. The role of overdiagnosis and reclassification in the marked increase of esophageal adenocarcinoma incidence. J Natl Cancer Inst. 2005;97:142-6.

2. Gamliel Z, Krasna MJ. Multimodality treatment of esophageal cancer. Surg Clin North Am. 2005;85:621-30.

3. Luketich JD, Alvelo-Rivera M, Buenaventura PO, Christie NA, McCaughan JS, Litle VR, et al. Minimally invasive esophagectomyoutcomes in 222 patients. Ann Surg. 2003;238:486-94

4. Swanson SJ, Batirel HF, Bueno R, Jaklitsch MT, Lukanich JM, Allred E, et al. Transthoracic esophagectomy with radical mediastinal and abdominal lymph node dissection and cervical esophagogastrostomy for esophageal carcinoma. Ann Thorac Surg. 2001;72:1918-24.

5. Chang JT, Katzka DA. Gastroesophageal reflux disease, Barrett esophagus, and esophageal adenocarcinoma. Arch Intern Med. 2004;164: $1482-8$.

6. Vizcaino AP, Moreno V, Lambert R, Parkin DM. Time trends incidence of both major histologic types of esophageal carcinomas in selected countries, 1973-1995. Int J Cancer. 2002;99:860-8.

7. Devesa SS, Blot WJ, Fraumeni JF Jr. Changing patterns in the incidence of esophageal and gastric carcinoma in the United States. Cancer. 1998; $83: 2049-53$

8. Lagergren J, Bergstrom R, Lindgren A, Nyren O. Symptomatic gastroesophageal reflux as a risk factor for esophageal adenocarcinoma. N Engl J Med. 1999;340:825-31.

9. Csendes A, Smok G, Burdiles P, Quesada F, Huertas C, Rojas J, et al. Prevalence of Barrett's esophagus by endoscopy and histologic studies: a prospective evaluation of 306 control subjects and 376 patients with symptoms of gastroesophageal reflux. Dis Esophagus. 2000;13:5-11.

10. Shaheen N, Ransohoff DF. Gastroesophageal reflux, barrett esophagus, and esophageal cancer: scientific review. JAMA. 2002;287:1972-81.

11. Altorki NK, Oliveria S, Schrump DS. Epidemiology and molecular biology of Barrett's adenocarcinoma. Semin Surg Oncol. 1997;13:270-80.

12. Falk GW, Rice TW, Goldblum JR, Richter JE. Jumbo biopsy forceps protocol still misses unsuspected cancer in Barrett's esophagus with high-grade dysplasia. Gastrointest Endosc. 1999;49:170-6.

13. Heitmiller RF, Redmond M, Hamilton SR. Barrett's esophagus with high-grade dysplasia. An indication for prophylactic esophagectomy. Ann Surg. 1996;224:66-71.

14. Nigro JJ, Hagen JA, DeMeester TR, DeMeester SR, Theisen J, Peters JH, et al. Occult esophageal adenocarcinoma: extent of disease and implications for effective therapy. Ann Surg. 1999;230:433-8.

15. Tharavej C, Hagen JA, Peters JH, Portale G, Lipham J, DeMeester SR, et al. Predictive factors of coexisting cancer in Barrett's high-grade dysplasia. Surg Endosc. 2006;20:439-43.

16. Larghi A, Lightdale CJ, Memeo L, Bhagat G, Okpara N, Rotterdam H. EUS followed by EMR for staging of high-grade dysplasia and early cancer in Barrett's esophagus. Gastrointest Endosc. 2005;62:16-23.

17. Sharma VK, Wang KK, Overholt BF, Lightdale CJ, Fennerty MB, Dean PJ, et al. Balloon-based, circumferential, endoscopic radiofrequency ablation of Barrett's esophagus: 1-year follow-up of 100 patients. Gastrointest Endosc. 2007;65:185-95.

18. Korst RJ, Altorki NK. High grade dysplasia: surveillance, mucosal ablation, or resection? World J Surg. 2003;27:1030-4.

19. Shaheen N, Ransohoff DF. Gastroesophageal reflux, Barrett esophagus, and esophageal cancer: clinical applications. JAMA. 2002;287:1982-6.

20. Reid BJ, Haggitt RC, Rubin CE, Roth G, Surawicz CM, Van Belle G, et al. Observer variation in the diagnosis of dysplasia in Barrett's esophagus. Hum Pathol. 1988;19:166-78.

21. Lee RC, Feinbaum RL, Ambros V. The $C$ elegans heterochronic gene lin-4 encodes small RNAs with antisense complementarity to lin-14. Cell. 1993;75:843-54.

22. Esquela-Kerscher A, Slack FJ. Oncomirs-microRNAs with a role in cancer. Nat Rev Cancer. 2006;6:259-69.

23. Lu J, Getz G, Miska EA, varez-Saavedra E, Lamb J, Peck D, et al. MicroRNA expression profiles classify human cancers. Nature. 2005;435: 834-8.

24. Calin GA, Ferracin M, Cimmino A, Di LG, Shimizu M, Wojcik SE, et al. A microRNA signature associated with prognosis and progression in chronic lymphocytic leukemia. $N$ Engl J Med. 2005;353:1793-801.

25. Iorio MV, Ferracin M, Liu CG, Veronese A, Spizzo R, Sabbioni S, et al. MicroRNA gene expression deregulation in human breast cancer. Cancer Res. 2005;65:7065-70.

26. Yanaihara N, Caplen N, Bowman E, Seike M, Kumamoto K, Yi M, et al. Unique microRNA molecular profiles in lung cancer diagnosis and prognosis. Cancer Cell. 2006;9:189-98.

27. Mattie MD, Benz CC, Bowers J, Sensinger K, Wong L, Scott GK, et al. Optimized high-throughput microRNA expression profiling provides novel biomarker assessment of clinical prostate and breast cancer biopsies. Mol Cancer. 2006;5:24.

28. Selaru FM, Zou T, Xu Y, Shustova V, Yin J, Mori Y, et al. Global gene expression profiling in Barrett's esophagus and esophageal cancer: a comparative analysis using cDNA microarrays. Oncogene. 2002;21: 475-8.

29. Helm J, Enkemann SA, Coppola D, Barthel JS, Kelley ST, Yeatman TJ. Dedifferentiation precedes invasion in the progression from Barrett's metaplasia to esophageal adenocarcinoma. Clin Cancer Res. 2005;11: 2478-85.

30. Watson DI, Wijnhoven BP, Michael MZ, Mayne GC, Hussey DJ. Hp24 microRNA expression profiles in Barrett's oesophagus. ANZ J Surg. 2007;77(Suppl 1):A45.

31. Bolstad BM, Irizarry RA, Astrand M, Speed TP. A comparison of normalization methods for high density oligonucleotide array data based on variance and bias. Bioinformatics. 2003;19:185-93.

32. Cameron AJ. Epidemiology of Barrett's esophagus and adenocarcinoma. Dis Esophagus. 2002;15:106-8. 
33. Wang S, Zhan M, Yin J, Abraham JM, Mori Y, Sato F, et al. Transcriptional profiling suggests that Barrett's metaplasia is an early intermediate stage in esophageal adenocarcinogenesis. Oncogene. 2006;25:3346-56.

34. Ramaswamy S, Tamayo P, Rifkin R, Mukherjee S, Yeang CH, Angelo M, et al. Multiclass cancer diagnosis using tumor gene expression signatures. Proc Natl Acad Sci U S A. 2001;98:15149-54.

35. He H, Jazdzewski K, Li W, Liyanarachchi S, Nagy R, Volinia S, et al. The role of microRNA genes in papillary thyroid carcinoma. Proc Natl Acad Sci U S A. 2005;102:19075-80.

36. Volinia S, Calin GA, Liu CG, Ambs S, Cimmino A, Petrocca F, et al. A microRNA expression signature of human solid tumors defines cancer gene targets. Proc Natl Acad Sci U S A. 2006;103:2257-61.

37. Chan JA, Krichevsky AM, Kosik KS. MicroRNA-21 is an antiapoptotic factor in human glioblastoma cells. Cancer Res. 2005;65: 6029-33.

38. Cheng AM, Byrom MW, Shelton J, Ford LP. Antisense inhibition of human miRNAs and indications for an involvement of miRNA in cell growth and apoptosis. Nucleic Acids Res. 2005;33:1290-7.

\section{Discussion}

Dr Nasser K. Altorki (New York, NY). Dr Litle, I congratulate you on a nice piece of work and nice presentation; I also congratulate your group on bringing this new field of microRNA expression profiles into the field of esophageal and other thoracic malignancies, particularly as related to using them as diagnostic tools, prognostic tools, and, most importantly I think, as tools for target discovery and mechanistically understanding better the biology. I think you have shown very nicely here that microRNA expression profiles can really distinguish histologies, AC versus SCC, and I think that you have also shown that the expression profiles can distinguish tumor from NSE.

From a mechanistic point of view, I do not know if you have had the opportunity to ascertain what the genomic sequence is that these microRNAs would target, or maybe even look at the putative signaling pathways that may be affected, for example, by mir-21, which, as I understand, is overexpressed in a variety of malignancies, both thoracic and extrathoracic, and has been found in at least one situation to target the p10 gene and its signaling pathway. I know you've had some frozen tissue. I don't know whether you were able to go back and examine those samples, or whether that lies in your future plans. That would be one question, the signaling pathways or the target sequences on the genome.
The other curiosity really relates to the 5 patients who had BE that appeared to have clustered either with squamous or $\mathrm{AC}$ or NSE, as I recall. Do you have any further follow-up with those patients to see whether those molecular signatures really translated into clinically significant outcomes?

Dr Litle. Thank you, Dr Altorki. With regard to the first question, our lab is primarily focused currently on biomarkers and detection of occult disease. We aren't involved in signal transduction research at this time, but certainly those are important questions. This is a relatively new field, and a lot is still not known about microRNAs in general. They are involved in cell differentiation, apoptosis, and proliferation, which emphasizes how little we know. Your questions will need to be answered with future studies.

Regarding the 5 patients with BE, we don't have any follow-up. The next step will be to validate the results with expression data. We have a bank of paraffin-embedded samples of dysplasia, BE specimens, and more AC specimens to add to our small sample here. We would use the microRNA expression profiles on a larger series and then design appropriate primers for reverse transcriptase-polymerase chain reaction so we could complete quantitative reverse transcriptase-polymerase chain reaction for patients with followup. Ideally, we would do follow-up on patients with BE-some with cancer and some not-so we could correlate the results. These research questions will be addressed in a newly funded National Institutes of Health grant.

Dr Thomas A. D'Amico (Durham, NC). How much tissue do you need to do the microRNA testing, and can you envisage a technique that wouldn't require endoscopy to get the same information?

Dr Litle. You mean to get the same information as far as whether someone has BE?

Dr D'Amico. Right. The goal is to take patients with BE or even just reflux and determine which ones are going to have BE or cancer. Can you envisage a way to do that without endoscopy?

Dr Litle. Use of serum markers for identifying BE in patients with reflux and cancer in patients with BE unfortunately doesn't appear to be available in the near future. We're not even at the point of being able to do this efficiently by endoscopic biopsy; we currently need enough tissue for both histologic and RNA analyses. Adequate endoscopic biopsy samples for molecular analysis of patients with $\mathrm{BE}$ would be our goal in the near future should our hypotheses prove true. 